\title{
Palm-based nonionic surfactants as emulsifiers for high internal phase emulsions.
}

\begin{abstract}
In the present study, a series of as-synthesized palm-based nonionic surfactants with various hydrophile-lipophile balance values were successfully synthesized. The critical micelle concentration and the Gibbs energy of the surfactants were determined and discussed. For the first time, the surfactants were used to stabilize three-component olein oil-in-water high internal phase emulsions, with an oil volume fraction of 0.85 , and which were easily prepared by one-pot homogenization. Proof of high stability was confirmed by the satisfactory rheological profiles and further enhanced by a three-month storage exercise at an elevated temperature which showed no significant physical and rheological changes. These results suggest that low concentration of the surfactants efficiently stabilized the emulsions with high content of oil. Based on the optical micrograph observation, an average droplet size of less than $10 \mu \mathrm{m}$ increased with increasing ethylene oxide chain length and temperature. The varying degree of viscosity resulted from the various ethylene oxide chain lengths of the surfactants. The hydration efficacy of the emulsions was examined in vivo using a corneometer. The impressive hydration efficacy of olein oil suggests that it could well be a potential moisturizing lipid which might interest dermatologists.
\end{abstract}

Keyword: High internal phase emulsion; Hydration; Olein oil; Palm-based surfactants; Rheology. 